\title{
Light-curing effects in acrylic-type dental nanocomposites probed by annihilating positrons: the case of loosely monolith Dipol ${ }^{\circledR}$ restoratives
}

\author{
Olha Shpotyuk $^{1} \cdot$ Adam $_{\text {Ingram }}^{2} \cdot$ Oleh Shpotyuk $^{3,4} \cdot$ Jozef Cebulski $^{5} \cdot$ Nina Smolar $^{1}$
}

Received: 30 November 2019 / Accepted: 2 January 2020 / Published online: 13 January 2020

(c) The Author(s) 2020

\begin{abstract}
The possibility of application of positron annihilation lifetime (PAL) spectroscopy to commercially available dimethacrylatebased dental restorative composites Dipol@ (Oksomat-AN Ltd, Ukraine) subjected to photopolymerization due to light curing is analyzed. The governing annihilation process in these composites is identified as mixed positron $\left(\mathrm{e}^{+}\right)$-positronium (Ps) trapping, where Ps decaying is caused entirely by input from free-volume holes in polymer matrix superimposed on free $\mathrm{e}^{+}$-trapping contribution from interfacial free-volume holes between filler nanoparticles and surrounded polymer matrix. Photopolymerization shrinkage is revealed through decrease in the average lifetime of annihilating positrons due to opposite changes in Ps-decaying and $\mathrm{e}^{+}$-trapping channels. The growing light-activated polymerization is characteristic of both intensities related to the second and third components in the unconstrained $\times 3$-term decomposed PAL spectra, accompanied by decrease in the corresponding lifetimes. This process resulted in enhanced trapping rate in the defects and depressed fractional free-volume saturation with light curing. Light exposure causes smaller voids in composites owing to free-volume fragmentation in Ps- and $\mathrm{e}^{+}$-trapping sites. The microstructure scenario for these transformations includes photo-induced cross-linking of structural chains in the polymer matrix, followed by conversion of o-Ps traps in interfacial free-volume voids near agglomerated filler nanoparticles. A meaningful description of this process is developed on the basis of the semiempirical model exploring the $\times 3-\times 2$-coupling decomposition algorithm.
\end{abstract}

Keywords Nanostructure $\cdot$ Nanoparticle $\cdot$ Light curing $\cdot$ Photopolymerization $\cdot$ Volumetric shrinkage $\cdot$ Filler-polymer composite

Jozef Cebulski

cebulski@ur.edu.pl

1 Danylo Halytsky Lviv National Medical University, 69, Pekarska st, Lviv, Ukraine

2 Opole University of Technology, 75, Ozimska str, 45370 Opole, Poland

3 Vlokh Institute of Physical Optics, 23, Dragomanov str, Lviv 79005, Ukraine

4 Jan Dlugosz University in Czestochowa, 13/15, al. Armii Krajowej, 42200 Czestochowa, Poland

5 Institute of Physics, University of Rzeszow, 1, Pigonia st, 35959 Rzeszow, Poland

\section{Background}

Progress in nowadays' biopolymer engineering depends on the reliable diagnostics of atomic-deficient spaces over the finest sub-nanometer length scales. One of such possibilities is related to positron annihilation lifetime (PAL) spectroscopy, the method probing the spacetime continuum relationships for interaction between electron and its antiparticle 
(positron) in structural entities such as free-volume defects, vacancies, vacancy-like clusters, interfacial voids, and pores (see, e.g., Jean 1990; Jean et al. 2013; Krause-Rehberg and Leipner 1999; Shpotyuk and Filipecki 2003).

This paper is intended to probe the PAL spectroscopy as an informative tool to parameterize free-volume evolution in light-cured dimethacrylate dental composites (DRC) exemplified by commercially available Dipol® (Dipol® 2012). This DRC is based on monomer consisting of bisphenol A-diglycidyl dimethacrylate (BisGMA) and triethyleneglycol dimethacrylate (TEGDMA) modified by multisized filler particles (dispersed silica glass with 2-0.1-0.02-0.003 $\mu \mathrm{m}$ particles). Because of weight-packing density at the level of $72 \%$, this nanocomposite belongs to loosely packed dental restoratives possessing respectively high volumetric shrinkage under photopolymerization above $2 \%$ like other DRC Charisma ${ }^{\circledR}$ (see, e.g., Shpotyuk et al. 2017).

\section{Methods}

\section{DRC preparation details}

The studied specimens of DRC Dipol@ (Dipol® 2012) were prepared by filling an inner volume of disc-shaped plastic molds of uniform size, $6 \mathrm{~mm}$ in diameter and $2 \mathrm{~mm}$ in thickness. The bottom end surface of the plastic disc was covered by polyethylene slice film, separated from the sample along with an outer ring around the disc before PAL experiments (this batch of non-polymerized DRC samples was marked as Dipol-0). Then, a part of these samples were polymerized by illuminating their upper surfaces with standard curing dental wireless LED source (LED.T4, SEASKY, China), which emitted light in the 420-480 nm spectral range with 900 $\mathrm{mW} / \mathrm{cm}^{2}$ output power density. To normalize the light-curing protocol for all DRC specimens, the end of the guide tip from the light source was maintained just above the sample surface at a distance of $7 \mathrm{~mm}$ so that the curing light beam fully covered the sample surface. The polymerization intervals with this LED source were 5, 20, 30, 40, 60 and $150 \mathrm{~s}$. The photopolymerized DRC specimens were stored dry at room temperature $\left(\sim 20^{\circ} \mathrm{C}\right)$ for 3 days prior to being used in further PAL measurements.

\section{PAL spectra measurement}

Experimental PAL spectra were registered with a fast-fast coincidence system of 230 ps resolution based on two Photonis XP2020/Q photomultiplier tubes coupled to $\mathrm{BaF}_{2}$ scintillator 25.4A10/2M-Q-BaF-X-N detectors (Scionix, Bunnik, Holland) and ORTEC® electronics (ORTEC, Oak
Ridge, TN, USA). To ensure most reliable measurements, each PAL spectrum was recorded at $T=22{ }^{\circ} \mathrm{C}$ and relative humidity of $35 \%$ in a normal measurement statistics (1 min of coincidences). The channel width of 6.15 ps allowed a total of 8000 channels. The radioactive ${ }^{22} \mathrm{Na}$ isotope of low $\sim 50 \mathrm{kBq}$ activity prepared from aqueous solution of ${ }^{22} \mathrm{NaCl}$ wrapped by Kapton ${ }^{\circledR}$ foil (DuPont ${ }^{\mathrm{TM}}$, Circleville, $\mathrm{OH}, \mathrm{USA}$ ) was used as the source of positrons sandwiched between the two tested samples.

The measured PAL spectra were processed with LT 9.0 program (Kansy 1996), stabilizing average positron lifetime $\tau_{a v} \sum_{v}$ as mass centrum of the PAL spectrum:

$\tau_{a v}^{\sum_{a v}}=\sum_{i} I_{i} \tau_{i}$,

where $\tau_{i}$ and $I_{i}$ denote the positron lifetime and intensity of the corresponding fitting components (the resultant accuracy in lifetime and intensity determination was $\pm 0.005 \mathrm{~ns}$ and $\pm 0.5 \%$, respectively).

\section{PAL spectra fitting}

The best fitting of PAL spectra was achieved via mixed channels of trapping due to defect-related positron $\mathrm{e}^{+}$traps and bound positron-electron $\mathrm{e}^{+}-\mathrm{e}^{-}$(positronium Ps) states. This task can be solved by fitting PAL spectra with three negative exponentials under unconstrained decomposition, because of repulsive interaction between positron and atomic nuclei of the environment, positron samples' intrinsic regions of minimal positive charge density, preferentially negativecharged or neutral free-volume voids. Describing positron trapping in terms of two-state model with only one kind of defect, the parameters of defect-free bulk lifetime $\tau_{\mathrm{b}}$, trapping rate in defects $\kappa_{\mathrm{d}}$ and fraction of trapped positrons $\eta$ can be simply calculated with respect to known formalism (see, e.g., Tuomisto and Makkonen 2013; Shpotyuk and Filipecki 2003). In addition, the difference between defect-related and defect-free positron lifetimes $\left(\tau_{2}-\tau_{\mathrm{b}}\right)$ can be accepted as a signature of the size of positron $\mathrm{e}^{+}$-traps in terms of equivalent number of vacancies (Krause-Rehberg and Leipner 1999).

Another channel is caused by positron annihilation from Ps state as free particles or interacting with an electron from the environment (see, e.g., Jean et al. 1990). In the ground state, Ps exists as para-Ps (p-Ps, antiparallel positron-electron spins) decaying intrinsically with two $\gamma$-quanta and character $0.125 \mathrm{~ns}$ lifetime in a vacuum, and ortho-Ps (o-Ps, parallel spins) decaying with three $\gamma$-quanta and 142 ns lifetime, these states being occupied with 1:3 ratio. In a matter, since positron wave function overlapping with electron outside, the annihilation with such electron having an antiparallel spin decreases lifetime to $0.5-10 \mathrm{~ns}$ resulting 
in two $\gamma$-rays ("pick-off" annihilation). The Ps localized in free-volume spaces gives indication of their mean radii $R$ in terms of long-lived $\tau_{3}$ lifetime (relative intensity of this component $I_{3}$ correlates with the density of Ps sites) with respect to Tao-Eldrup equation:

$\tau_{3}=0.5 \cdot\left[1-\frac{R}{R+\Delta R}+\frac{1}{2 \pi} \cdot \sin \left(\frac{2 \pi R}{R+\Delta R}\right)\right]^{-1}$,

where $\Delta R=0.166 \mathrm{~nm}$ is the fitted empirical layer thickness (Jean et al. 1990).

By fitting Eq. (2) with measured $\tau_{3}$ values, the radius $R_{3}$ and the respective spherical free volumes $V_{\mathrm{f}}$ can be determined, giving the possibility to calculate the fractional free volume $f_{\mathrm{v}}{ }^{3}=C \cdot I_{3} \cdot V_{\mathrm{f}}$ using the empirical constant $C=0.0018 \AA^{-3}$ (Jean et al. 2013).

\section{PAL spectra treatment within $\times 3-\times 2-C D A$}

In highly inhomogeneous substances such as DRC, the PAL spectra are composed through mixed $\mathrm{e}^{+}$-Ps trapping (Shpotyuk et al. 2016,2017). Assuming that changes in o-Ps and $\mathrm{e}^{+}$-trapping sites under light curing are interconnected so that no effects occur in other annihilation channels (such as annihilation from defect-free bulk states, or generation of Ps- or $\mathrm{e}^{+}$traps), we can treat the measured $\times 3$-component PAL spectra within $\times 3-\times 2-$ CDA (coupling decomposition algorithm). In this approach (see Shpotyuk et al. 2015; Shpotyuket al. 2016), the experimental $\times 3$-term reconstructed PAL spectra are transformed to generalized $\times 2$-term form for host (non-polymerized) and modified (photopolymerized or light-cured) DRC, the second component involving contributions from all possible trapping sources. Such simplification allows resolving additional component in the generalized $\times 2$-term PAL spectrum for modified matrix (with lifetime $\tau_{\text {int }}$ and intensity $\left.I_{\text {int }}\right)$, the compensating $\left(\tau_{\mathrm{n}}\right.$, $I_{\mathrm{n}}$ ) input in the first channel being found assuming a reasonable condition of full inter-channel equilibrium (Shpotyuk et al. 2015). The parameterization of transformed Ps- $\mathrm{e}^{+}$traps in photopolymerized DRC matrix can be performed accepting $\left(\tau_{\mathrm{n}}, I_{\mathrm{n}}\right)$ and $\left(\tau_{\mathrm{int}}, I_{\mathrm{int}}\right)$ as the respective first and second components of the generalized $\times 2$-term PAL spectrum for some hypothetic medium obeying parameterization with respect to formalism of a simple two-state positron trapping model (Tuomisto and Makkonen 2013). Defect-related $\tau_{\text {int }}$ lifetime in this model reflects appearing/disappearing traps in dependence on positive/negative sign of $I_{\mathrm{n}}$ and $I_{\text {int }}$ intensities (Shpotyuk et al. 2015).

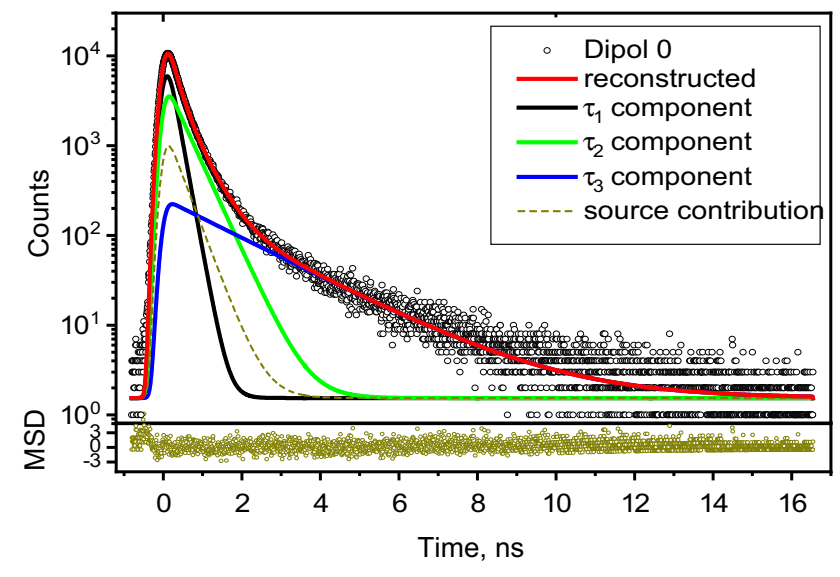

Fig. 1 Raw PAL spectra of non-polymerized DRC Dipol® reconstructed from unconstrained $\times 3$-fitting at the background of source contribution (bottom insets show statistical scatter of variance)

\section{Results and discussion}

The raw PAL spectra of non-polymerized DRC Dipol ${ }^{\circledR}$ (Dipol-0 specimen) is shown in Fig. 1. Similar spectra were collected for other samples subjected to photopolymerization through the light-curing procedure. These PAL spectra were unambiguously reconstructed from an unconstrained $\times 3$-term fitting route. The narrow-restricted statistical scatter of variance tightly grouped around the 0 -axis on Fig. 1 testifies that PAL measurements was adequately described within this decomposition. The respective best-fit parameters, $\mathrm{e}^{+}$-trapping and Ps-decaying modes are shown in Tables 1 and 2.

With respect to the PAL data parameterized within unconstrained $\times 3$-term analysis (Tables 1,2 ), the annihilation process in all DRC Dipol ${ }^{\circledR}$ samples can be identified as mixed $\mathrm{e}^{+}$-Ps trapping, where "pure" o-Ps decaying is caused entirely by input from free-volume holes in the polymer matrix (third component), while the second component is defined mainly by cumulative input of free $\mathrm{e}^{+}$-trapping sites in a filler (including interfacial free-volume holes between filler nanoparticles). Typical radii of o-Ps-traps in this DRC estimated from Eq. (2) approach 2.5-2.9 $\AA$. The same applies to $\mathrm{e}^{+}$-trapping channel defined by defect lifetimes $\tau_{2} \cong 0.40-0.44 \mathrm{~ns}$ (strict parameterization of this channel in terms of two-state trapping model is rather meaningless under essential input from o-Ps decaying).

It is known that light curing causes volumetric shrinkage in polymer-filler composites, with this macroscopic sensitive parameter approaching $2.2 \%$ for DRC Dipol®. Due to trapping parameters defined from $\times 3$-component best-fitted PAL spectra (Table 1), photopolymerization shrinkage in the studied DRC is well revealed through decrease in average lifetime of annihilating positrons $\tau_{\text {av }}$ (from $0.466 \mathrm{~ns}$ in Dipol-0 to $0.442 \mathrm{~ns}$ in Dipol-60, i.e. on 5.2\%), this effect 
being caused by mutually opposite changes in the o-Ps and $\mathrm{e}^{+}$-trapping channels. The photopolymerized DRC possess reduced long-lived $\tau_{3}$ lifetimes, but increased $I_{3}$ intensities, thus resulting in smaller fractional free volumes $f_{\mathrm{v}}$ (on $\sim 22 \%$ ). At the same time, the fraction of trapped positrons $\eta$ shows overbalanced tendency with light curing due to increase of $\sim 15 \%$ (Table 2 ). These parameters change monotonically with photoexposure tending to saturation after $60 \mathrm{~s}$ of light curing.

Changes in Ps-decaying modes can be ascribed preferentially to cross-linking of structural chains dominating in the "pure" polymer matrix, like it occurs in other polymers under UV light exposure (Ramani and Ranganathaiah, 2000), thermal curing (Srithawatpong et al. 1999) or $\gamma$-irradiation (Hyla et al. 2005). In fact, this photoinduced process causes smaller voids in DRC owing to overall free-volume fragmentation in both the o-Ps and $\mathrm{e}^{+}$-trapping sites. In the vicinity of agglomerated filler particles, the fragmented o-Ps traps convert into interfacial voids (triple junctions or pseudogap holes at the interface between the outer surface layer of the filler particles and the innermost layer of the surrounding polymer matrix), which are also efficient trapping sites for positrons (corresponding to increased fraction of trapped positrons $\eta$ in light-cured DRC).

By assuming full conversion of o-Ps trapping sites in interfacial $\mathrm{e}^{+}$-traps (ignoring void fragmentation without changing in trapping type), we can treat these data within $\times 3-\times 2$-CDA (Shpotyuk et al. 2015,2016). Due to the preferential disappearance of free-volume voids under light-curing polymerization in polymer-filler composites, this mathematical treatment of experimental $\times 3$-term decomposed PAL spectra is convenient to perform for uncured samples with respect to light-cured ones (in this case, both $I_{\mathrm{n}}$ and $I_{\text {int }}$ intensities are positive).

The PAL trapping parameters for non-polymerized Dipol-0 sample with respect to light-cured DRC Dipol® probes using $\times 3-\times 2-$ CDA are presented in Table 3 . Since $\tau_{\text {int }}$ values (approaching $0.6-0.8 \mathrm{~ns}$ ) exceed the
Table 1 The best-fit PAL spectra parameters for DRC Dipol ${ }^{\circledR}$ determined within unconstrained $\times 3$-term decomposition procedure

Table 2 The best-fit PAL spectra trapping modes for DRC Dipol® determined within unconstrained $\times 3$-term decomposition procedure ignoring o-Ps-decaying contribution

Table 3 The calculated $\mathrm{e}^{+}$-trapping modes for nonpolymerized Dipol-0 in respect to light-cured DRC Dipol® samples within $\times 3-\times 2-\mathrm{CDA}$

\begin{tabular}{llllllll}
\hline DRC, exposure (s) & [FIT-1] & $\tau_{1}, \mathrm{~ns}$ & $\tau_{2}, \mathrm{~ns}$ & $\tau_{3}, \mathrm{~ns}$ & $I_{2}$, a.u & $I_{3}$, a.u & $\tau_{\text {av. }}, \mathrm{ns}$ \\
\hline Dipol-0 & 0.038 & 0.178 & 0.444 & 1.978 & 0.510 & 0.085 & 0.466 \\
Dipol-5 & 0.004 & 0.164 & 0.424 & 1.723 & 0.530 & 0.096 & 0.451 \\
Dipol-20 & 0.031 & 0.165 & 0.421 & 1.668 & 0.530 & 0.101 & 0.452 \\
Dipol-30 & 0.044 & 0.160 & 0.406 & 1.601 & 0.540 & 0.104 & 0.444 \\
Dipol-40 & 0.024 & 0.160 & 0.415 & 1.606 & 0.540 & 0.104 & 0.446 \\
Dipol-60 & 0.034 & 0.155 & 0.403 & 1.601 & 0.560 & 0.103 & 0.442 \\
Dipol-150 & 0.055 & 0.159 & 0.411 & 1.598 & 0.560 & 0.105 & 0.451 \\
\hline
\end{tabular}

\begin{tabular}{|c|c|c|c|c|c|c|c|c|c|}
\hline \multirow[t]{2}{*}{ DRC, exposure (s) } & \multicolumn{6}{|c|}{$\mathrm{e}^{+}$-trapping modes } & \multicolumn{3}{|c|}{ Ps-decaying modes } \\
\hline & $\tau_{\text {av. }}{ }^{\mathrm{tr}}, \mathrm{ns}$ & $\tau_{\mathrm{b}}, \mathrm{ns}$ & $\kappa_{\mathrm{d}}, \mathrm{ns}^{-1}$ & $\tau_{2}-\tau_{\mathrm{b}}, \mathrm{ns}$ & $\tau_{2} / \tau_{\mathrm{b}}$, a.u & $\eta$, a.u & $R_{3}, \mathrm{~nm}$ & $V_{\mathrm{f}}, 10^{-3} \mathrm{~nm}^{3}$ & $f_{\mathrm{v}}^{3}, \%$ \\
\hline Dipol-0 & 0.325 & 0.266 & 1.86 & 0.178 & 1.67 & 0.33 & 0.286 & 98 & 1.50 \\
\hline Dipol-5 & 0.316 & 0.256 & 2.19 & 0.168 & 1.65 & 0.36 & 0.258 & 72 & 1.24 \\
\hline Dipol-20 & 0.316 & 0.257 & 2.16 & 0.164 & 1.64 & 0.36 & 0.255 & 69 & 1.26 \\
\hline Dipol-30 & 0.309 & 0.253 & 2.31 & 0.153 & 1.60 & 0.37 & 0.253 & 68 & 1.19 \\
\hline Dipol-40 & 0.312 & 0.253 & 2.29 & 0.162 & 1.64 & 0.37 & 0.249 & 65 & 1.20 \\
\hline Dipol-60 & 0.309 & 0.251 & 2.45 & 0.152 & 1.61 & 0.38 & 0.248 & 64 & 1.17 \\
\hline Dipol-150 & 0.317 & 0.259 & 2.44 & 0.152 & 1.59 & 0.38 & 0.248 & 64 & 1.20 \\
\hline
\end{tabular}

\begin{tabular}{|c|c|c|c|c|c|c|c|c|c|}
\hline \multirow[t]{2}{*}{ DRC system } & \multicolumn{2}{|c|}{ I component } & \multicolumn{2}{|c|}{ II component } & \multicolumn{5}{|c|}{$\mathrm{e}^{+}$-trapping modes } \\
\hline & $\tau_{\mathrm{n}}, \mathrm{ns}$ & $I_{\mathrm{n}}$, a.u & $\tau_{\mathrm{int}}, \mathrm{ns}$ & $I_{\text {int }}$, a.u & $\tau_{\mathrm{av}}, \mathrm{ns}$ & $\tau_{\mathrm{b}}, \mathrm{ns}$ & $\kappa_{\mathrm{d}}, \mathrm{ns}^{-1}$ & $\tau_{2}-\tau_{\mathrm{b}}, \mathrm{ns}$ & $\tau_{2} / \tau_{\mathrm{b}}$, a.u \\
\hline Dipol-0-Dipol-5 & 0.473 & 0.018 & 0.674 & 0.041 & 0.613 & 0.579 & 0.440 & 0.077 & 1.13 \\
\hline Dipol-0-Dipol-20 & 0.290 & 0.041 & 0.604 & 0.064 & 0.482 & 0.424 & 1.097 & 0.180 & 1.42 \\
\hline Dipol-0-Dipol-30 & 0.313 & 0.046 & 0.686 & 0.069 & 0.537 & 0.464 & 1.041 & 0.224 & 1.48 \\
\hline Dipol-0-Dipol-40 & 0.344 & 0.039 & 0.630 & 0.069 & 0.527 & 0.485 & 0.847 & 0.146 & 1.30 \\
\hline Dipol-0-Dipol-60 & 0.586 & 0.021 & 0.840 & 0.048 & 0.762 & 0.742 & 0.359 & 0.098 & 1.13 \\
\hline Dipol-0-Dipol-150 & 0.394 & 0.031 & 0.708 & 0.057 & 0.597 & 0.552 & 0.725 & 0.156 & 1.28 \\
\hline
\end{tabular}


characteristic level ( $0.5 \mathrm{~ns})$ of Ps self-annihilation in a vacuum (Jean et al. 2013), the hypothetical medium formed in these samples under photopolymerization can be imagined as rather loose substances possessing a wide range of o-Ps trapping sites. These free-volume holes (triple junctions in the filler-polymer interface) with radii $0.13 \mathrm{~nm}$ estimated with respect to Eq. (2) disappear under light curing from DRC Dipol ${ }^{\circledR}$ at a cost of interfacial positron traps having $\tau_{3} \approx 1.6 \mathrm{~ns}$ in the polymer part of the matrix near agglomerated filler nanoparticles.

\section{Conclusions}

The characterization possibilities of PAL spectroscopy are analyzed for application to commercially available acrylic-type dental restorative composite Dipol ${ }^{\circledR}$ (Dipol ${ }^{\circledR}$ 2012) subjected to light curing from LED source emitted with $\sim 900 \mathrm{~mW} / \mathrm{cm}^{2}$ power density $(420-480 \mathrm{~nm})$.

With respect to the PAL data parameterized within unconstrained $\times 3$-term analysis, the governing annihilation process in these composites is identified as mixed positron $\left(\mathrm{e}^{+}\right)$-positronium (Ps) trapping, where Ps-decaying is caused entirely by input from free-volume holes in the polymer matrix, while the second component is defined by free $\mathrm{e}^{+}$-trapping in interfacial free-volume holes between the filler nanoparticles and the surrounding polymer matrix. The photopolymerization shrinkage is revealed through decrease in the average lifetime of annihilating positrons due to mutually opposite changes in Ps-decaying and $\mathrm{e}^{+}$-trapping channels. The growing light-activated polymerization is characteristic of both intensities related to the second and third components in the $\times 3$-term decomposed PAL spectra accompanied by decrease in the corresponding positron lifetimes. This process results in enhanced trapping rate in defects and depressed fractional free volume saturated with light curing. Light exposure causes smaller voids in composites owing to free-volume fragmentation in Ps- and $\mathrm{e}^{+}$-trapping sites. The plausible microstructure scenario for these transformations includes photo-induced cross-linking of structural chains in the polymer matrix, followed by conversion of o-Ps traps in interfacial free-volume voids in the vicinity of agglomerated filler nanoparticles.

Meaningful phenomenological description of this process in Dipol ${ }^{\circledR}$ composites can be developed on the basis of semi-empirical model exploring the $\times 3-\times 2$-coupling decomposition algorithm.

\section{Compliance with ethical standards}

Conflict of interest On behalf of all authors, the corresponding author states that there is no conflict of interest.
Open Access This article is licensed under a Creative Commons Attribution 4.0 International License, which permits use, sharing, adaptation, distribution and reproduction in any medium or format, as long as you give appropriate credit to the original author(s) and the source, provide a link to the Creative Commons licence, and indicate if changes were made. The images or other third party material in this article are included in the article's Creative Commons licence, unless indicated otherwise in a credit line to the material. If material is not included in the article's Creative Commons licence and your intended use is not permitted by statutory regulation or exceeds the permitted use, you will need to obtain permission directly from the copyright holder. To view a copy of this licence, visit http://creativecommons.org/licenses/by/4.0/.

\section{References}

Dipol $^{\circledR}$ (2012) Composite Universal. Instruction on using Dipol materials. Oksomat-AN. Ukraine Dental Products, Kyiv

Hyla M, Filipecki J, Swiatek J, Mervinskii RI (2005) Gamma irradiation effects on UV-cured polymers based on acrylate oligomers studied by positron annihilation lifetime spectroscopy. J NonCryst Solids 351:1473-1476. https://doi.org/10.1016/j.jnoncrysol .2005 .03 .031

Jean YC (1990) Positron annihilation spectroscopy for chemical analysis: a novel probe for microstructural analysis of polymers. Microchem J 42:72-102. https://doi.org/10.1016/0026-265X(90)90027 $-3$

Jean YC, Van Horn JD, Hung WS, Lee KR (2013) Perspective of positron annihilation spectroscopy in polymers. Macromoleculs 46:7133-7145. https://doi.org/10.1021/ma401309x

Kansy J (1996) Microcomputer program for analysis of positron annihilation lifetime spectra. Nucl Instr Methods Phys Res A 374:235244. https://doi.org/10.1016/0168-9002(96)00075-7

Krause-Rehberg R, Leipner HS (1999) Positron annihilation in semiconductors. Defect studies. Springer-Verlag, Berlin-Heidelberg

Ramani R, Ranganathaiah C (2000) Degradation of acrylonitrile-butadiene-styrene and polycarbonate by UV irradiation. Polym Degrad Stabil 69:347-354. https://doi.org/10.1016/S0141-3910(00)00081 $-1$

Shpotyuk O, Filipecki J (2003) Free volume in vitreous chalcogenide semiconductors: possibilities of positron annihilation lifetime study. Wydaw. Wyższej Szkoły Pedagogicznej, Czestochowa

Shpotyuk O, Filipecki J, Ingram A, Golovchak R, Vakiv M, Klym H, Balitska V, Shpotyuk M, Kozdras A (2015) Positronics of subnanometer atomistic imperfections in solids as a high-informative structure characterization tool. Nanoscale Res Lett. https://doi. org/10.1186/s11671-015-0764-Z

Shpotyuk O, Ingram A, Filipecki J, Bujňáková Z, Baláž P (2016a) Positron annihilation lifetime study of atomic imperfections in nanostructurized solids: on the parameterized trapping in wet-milled arsenic sulfides $\mathrm{As}_{4} \mathrm{~S}_{4}$. Phys Status Solidi B 253:1054-1059. https ://doi.org/10.1002/pssb.201552560

Shpotyuk O, Ingram A, Shpotyuk O (2016b) Free volume structure of acrylic-type dental nanocomposites tested with annihilating positrons. Nanoscale Res. Lett. 11:528. https://doi.org/10.1186/ s11671-016-1751-8

Shpotyuk O, Ingram A, Shpotyuk O, Bezvushko E (2017) Light-cured dimethacrylate dental restorative composites under a prism of annihilating positrons. Polim. Med. 47:91-100https://doi. org/10.17219/pim/81450

Srithawatpong R, Peng ZL, Olson BG, Jamieson AM, Simha R, McGervey JD, Maier TR, Halasa AF, Ishida H (1999) Positron annihilation lifetime studies of changes in free volume on crosslinking cis-polyisoprene, high-vinyl polybutadiene, and their miscible blends. J Polym Sci Pol Phys 37:2754-2770. https://doi.

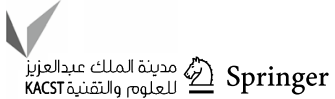


org/10.1002/(SICI)1099-0488(19991001)37:19\%3c2754:AIDPOLB5\%3e3.0.CO;2-F

Tuomisto F, Makkonen I (2013) Defect identification in semiconductors with positron annihilation: experiment and theory. Rev
Mod Phys 85:1583-1631. https://doi.org/10.1103/RevModPhys .85 .1583 